\title{
Expression of DNA Methyltransferase 1 Is a Hallmark of Melanoma, Correlating with Proliferation and Response to B-Raf and Mitogen-Activated Protein Kinase Inhibition in Melanocytic Tumors
}

\author{
Maximilian Gassenmaier, ${ }^{*}$ Maximilian Rentschler, ${ }^{*}$ Birgit Fehrenbacher, ${ }^{*}$ Thomas K. Eigentler, ${ }^{*}$ Kristian Ikenberg, ${ }^{\dagger}$ \\ Corinna Kosnopfel, ${ }^{*}$ Tobias Sinnberg, ${ }^{*}$ Heike Niessner, ${ }^{*}$ Hans Bösmüller, ${ }^{\ddagger}$ Nikolaus B. Wagner, ${ }^{\S}$ Martin Schaller, ${ }^{*}$ Claus Garbe, ${ }^{*}$ \\ and Martin Röcken*
}

From the Department of Dermatology, ${ }^{*}$ and the Institute of Pathology, ${ }^{\ddagger}$ Eberhard Karls University of Tübingen, Tübingen, Germany; the Institute of Pathology and Molecular Pathology, ${ }^{\dagger}$ University Hospital Zurich, Zurich; and the Department of Dermatology, ${ }^{\S}$ Cantonal Hospital St. Gallen, St. Gallen, Switzerland

\author{
Accepted for publication \\ July $3,2020$. \\ Address correspondence to \\ Maximilian Gassenmaier, \\ M.D., Department of Derma- \\ tology, Eberhard Karls Univer- \\ sity of Tübingen, \\ Liebermeisterstr. 25, Tübingen \\ 72076, Germany. E-mail: \\ maximilian.gassenmaier@med. \\ uni-tuebingen.de.
}

\begin{abstract}
Aberrant DNA methylation is an epigenetic hallmark of melanoma, but the expression of DNA methyltransferase (Dnmt)-1 in melanocytic tumors is unknown. Dnmt1 expression was analyzed in primary melanocytes, melanoma cell lines, and 83 melanocytic tumors, and its associations with proliferation, mutational status, and response to B-Raf and mitogen-activated protein kinase kinase (MEK) inhibition were explored. Dnmt1 expression was increased incrementally from nevi [mean fluorescence intensity (MFI), 48.1; interquartile range, 41.7 to 59.6] to primary melanomas (MFI, 68.8; interquartile range, 58.4 to 77.0$)$ and metastatic melanomas (MFI, 87.5; interquartile range, 77.1 to 114.5$)(P<0.001)$. Dnmt1 expression was correlated with Ki-67 expression (Spearman correlation, $0.483 ; P<0.001$ ) and was independent of $B R A F$ mutation status $(P=0.55)$. In BRAF-mutant melanoma, Dnmt1 was downregulated during response to $B$-Raf and MEK inhibition and was again up-regulated on drug resistance in vitro and in vivo. Degradation of Dnmt1 by the histone deacetylase inhibitor suberoylanilide hydroxamic acid was associated with decreased cell viability in B-Raf inhibitor-sensitive and -resistant cell lines. This study demonstrates that Dnmt1 expression is correlated with proliferation in melanocytic tumors, is increased with melanoma progression, and is associated with response to B-Raf and MEK inhibition. Given its strong expression in metastatic melanoma, Dnmt1 may be a promising target for combined epigenetic and immunotherapy. (Am J Pathol 2020, 190: 2155-2164; https:// doi.org/10.1016/j.ajpath.2020.07.002)
\end{abstract}

Epigenetic modifications refer to heritable changes in gene expression that occur independent of changes in the primary DNA sequence. ${ }^{1}$ DNA methylation is an essential component of epigenetic modification and provides a stable genesilencing mechanism that plays an important role in regulating gene expression and chromatin architecture. ${ }^{1}$ In mammals, DNA methylation occurs primarily by the covalent addition of methyl groups at cytosine residues in $\mathrm{CpG}$ dinucleotides. Site-specific hypermethylation can contribute to tumor development and progression by silencing tumor suppressor genes and has been found in many cancers. ${ }^{1,2}$
There are significant differences in methylation patterns between melanocytic nevi and melanoma, highlighting that aberrant DNA methylation is an epigenetic hallmark of melanoma. $^{3-5}$ DNA methyltransferase (Dnmt)-1 is the major enzyme involved in maintaining methylation patterns after cellular replication, but its expression in melanocytic tumors in

Supported in part by Novartis Pharma GmbH project S2TAF-015 (M.G.).

M.G. and M.R. contributed equally to this work.

Disclosures: None declared. 
unknown. ${ }^{6}$ Clinical trials of hypomethylating drugs that target Dnmt1 in patients with metastatic melanoma are currently being conducted, and these drugs have recently shown promising immunomodulatory and antitumor activity. ${ }^{7}$ Dnmt 1 depletion has been reported to overcome resistance of interferon-induced apoptosis in melanoma cells, to lead to reactivation of cyclin-dependent kinase inhibitor $2 \mathrm{~A}$ protein in cancer cells, and to trigger senescence in fibroblasts. ${ }^{8-11}$ As interferon- $\gamma$ has been associated with the stable arrest and induction of a senescence phenotype in cancers in vivo and melanoma cells in vitro, ${ }^{12-14}$ the role of Dnmt 1 in melanocytic tumors was investigated in the present study.

In this study, Dnmt1 expression was strongly correlated with the proliferation of melanocytic tumors and was associated with the response of melanoma cells to B-Raf and mitogen-activated protein kinase kinase (MEK) inhibitors. Finally, the translational and therapeutic implications of the findings are discussed.

\section{Materials and Methods}

\section{Statistical Analysis}

Statistical calculations were performed with SPSS statistics software version 23.0 (IBM, Armonk, NY). Numeric variables are described by median values and interquartile ranges (IQRs) or means \pm SD. Graphs were built with JMP software version 14.2.0 (SAS, Cary, NC) and Prism software version 8.4 (GraphPad, San Diego, CA). Throughout the analysis, $P$ values of $<0.05$ were considered as statistically significant.

\section{Cell Culture}

Melanoma cell lines were cultured in RPMI 1640 medium (Thermo Fisher Scientific, Waltham, MA), which was supplemented with 10\% fetal calf serum (Merck, Darmstadt, Germany) and $1 \%$ penicillin/streptomycin (Thermo Fisher Scientific).

Melanoma cell lines were kindly provided by Meenhard Herlyn (451LU, 1205LU, SbCl2, WM35, WM793, WM1346, WM1366, WM1552, WM115, and Mel1617), by Léon C. van Kempen (MV3), and by Claus Garbe (SKMel19) or were purchased from the ATCC (Manassas, VA; SKMel28, A375, and MeWo). Vemurafenib-resistant cell lines were generated by continuous treatment with an increasing concentration of vemurafenib, up to $2 \mu \mathrm{mol} / \mathrm{L}$, for several months. ${ }^{15}$ The effect of targeted therapy on Dnmt 1 expression in vitro was evaluated after treatment with $2 \mu \mathrm{mol} / \mathrm{L}$ vemurafenib and/or $0.1 \mu \mathrm{mol} / \mathrm{L}$ cobimetinib or $2 \mu \mathrm{mol} / \mathrm{L}$ suberoylanilide hydroxamic acid (SAHA) for 24 hours. Stock solutions of the B-Raf inhibitor (BRafi) vemurafenib (LC Laboratories, Woburn, MA), the MEK inhibitor (MEKi) cobimetinib (Hycultec, Beutelsbach, Germany), and the histone deacetylase inhibitor SAHA (InvivoGen, San Diego, CA) were prepared in dimethyl sulfoxide. Cell viability was assessed using the 4-methylumbelliferyl heptanoate assay. In brief, $2.5 \times 10^{3}$ cells were seeded into 96-well plate cavities in six replicates 24 hours before treatment with vemurafenib or SAHA (both up to $25 \mu \mathrm{mol} / \mathrm{L}$ for 72 hours). Viability was analyzed after a washing step with phosphate-buffered saline and subsequent incubation of the cells in $100 \mu \mathrm{g} / \mathrm{mL}$ 4-methylumbelliferyl heptanoate (Merck) diluted in phosphate-buffered saline for 1 hour at $37^{\circ} \mathrm{C}$. The fluorescence $(\mathrm{ex}=355 \mathrm{~nm}$, em $=460 \mathrm{~nm})$ was detected in a Tristar fluorescence microplate reader (Berthold Technologies, Bad Wildbad, Germany).

Human primary melanocytes were isolated from human foreskin samples as described ${ }^{16}$ and cultivated in Dermalife M medium (Cell Systems, Troisdorf, Germany). Melanocytes were expanded until confluency.

\section{Western Blot Analysis}

Cells were lysed in RIPA buffer $(50 \mathrm{mmol} / \mathrm{L}$ Tris- $\mathrm{HCl}$, $\mathrm{pH} 8.0$, $150 \mathrm{mmol} / \mathrm{L} \mathrm{NaCl}, 1 \% \mathrm{NP}-40,0.5 \%$ sodium deoxycholate, $0.1 \%$ SDS) containing a protease inhibitor cocktail and a phosphatase inhibitor cocktail (cOmplete and PhosSTOP; Roche Diagnostics, Rotkreuz, Switzerland). After the determination of protein content by the Pierce BCA protein assay kit (Thermo Fisher Scientific), proteins were resolved by $10 \%$ SDS-PAGE, transferred onto an Immobilon-FL PVDF transfer membrane (Merck), and blocked with Odyssey or Intercept blocking buffer (LI-COR Biosciences, Lincoln, NE). The membrane was then incubated overnight at $4{ }^{\circ} \mathrm{C}$ with primary antibodies anti- $\beta$-actin (1:5000; catalog number MAB1501R; Merck) and anti-Dnmt1 (1:500; catalog number ab13537; Abcam, Cambridge, UK), or anti- $\beta$-actin (1:1000; catalog number 4970; Cell Signaling Technology, Danvers, MA) and anti-proliferating cell nuclear antigen (PCNA; 1:2000; catalog number 2586; Cell Signaling Technology), respectively. After washing with Tris-buffered saline with Tween 20 and subsequent blocking, the blots were incubated for 1 hour at room temperature with fluorescent dye-conjugated secondary antibodies IRDye $800 \mathrm{CW}$ goat anti-mouse IgG $(1: 15,000$; cata$\log$ number 926-32210) and IRDye 680RD goat anti-rabbit IgG (1:20,000; catalog number 926-68071) (both, LI-COR Biosciences) and washed again. The antibody binding was then detected with the Odyssey Sa Imager (LI-COR Biosciences). Immunoreactive bands were quantified using Image Studio Lite software version 3.1 (LI-COR Biosciences), and the target/reference protein ratio of the samples was calculated.

\section{Gene Expression Analysis}

Gene expression information was retrieved from publicly accessible data sets and analyzed with the web-based genomics analysis and visualization platform R2 (https:// hgserverl.amc.nl/cgi-bin/r2/main.cgi, last accessed March 30, 2020). In each analysis, DNMTI expression values were centered around 0 ( $\log 2$ centered). The following data sets from Gene Expression Omnibus (http://www.ncbi.nlm.nih. gov/geo) were used: accession numbers GSE3189 (nevi, $n=18$; melanoma, $n=45)^{17}$ and GSE116237 (patient- 
derived xenograft model under treatment with dabrafenib and trametinib; cells analyzed pretreatment, $n=172$; 4 days on treatment, $n=155$; 28 days on treatment, $n=199$; and 57 days on treatment, $n=148)$. ${ }^{18}$

\section{IHC and Immunofluorescence Analysis}

Formalin-fixed, paraffin-embedded (FFPE) tissue was cut into $2.5-\mu \mathrm{m}-$ thick sections, deparaffinized, and stained with an anti-Dnmt1 antibody (1:1500; catalog number ab13537; Abcam) on an automated immunostainer (Leica Bond-MAX; Leica Biosystems, Wetzlar, Germany). For immunofluorescence, the following antibodies were used: anti-Dnmt1 (1:100; catalog number ab19905; Abcam), anti-melanoma (1:200; catalog number ab733; Abcam), anti-Ki-67 (1:75; catalog number 14-5698; Thermo Fisher Scientific), Alexa Fluor 488 donkey anti-mouse (1:250; catalog number 715-546-151; Dianova, Hamburg, Germany), Alexa Fluor 647 donkey antirat (1:250; catalog number 712-606-153; Dianova), and Cy3 donkey anti-rabbit (1:250; catalog number 711-166-152; Dianova). Nuclei were stained with DAPI (1:2000; catalog number D9542; Sigma-Aldrich/Merck). Immunofluorescence images were obtained with a confocal laser scanning microscope (LSM 800; Carl Zeiss, Jena, Germany), and images were processed and analyzed with ZEN Blue software version 2.3 (Carl Zeiss). At least two representative areas of each melanocytic tumor were examined under the microscope at $250 \times$ or $400 \times$ magnification, with a median (IQR) of 268 (143 to 450) cells analyzed. The nuclear mean fluorescence intensity values of Dnmt1 and Ki-67 in anti-melanoma antibody-positive cells were measured in a computerized manner (Supplemental Figure S1 and Supplemental Table S1) and were calculated
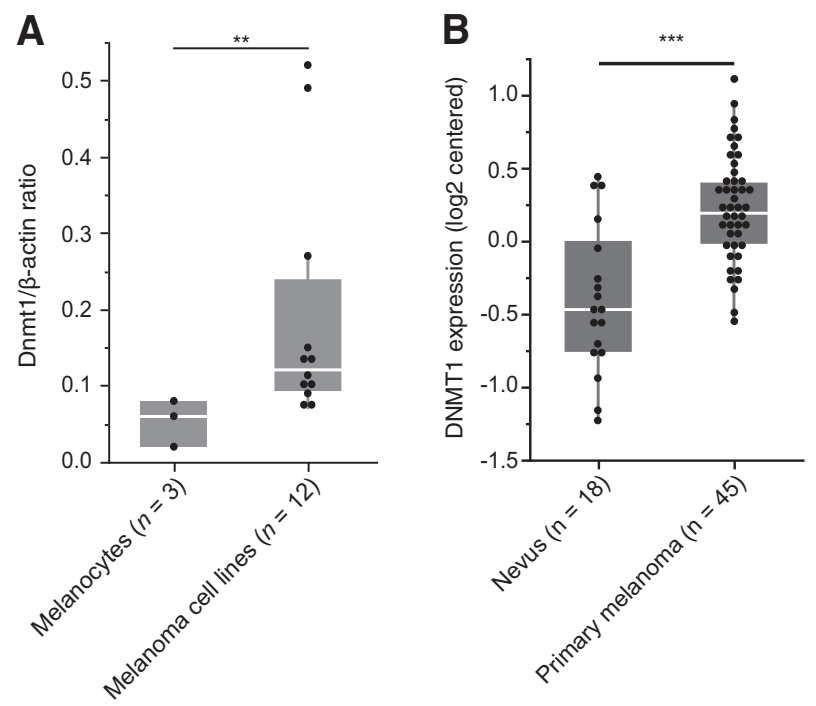

Figure 1 DNA methyltransferase 1 (Dnmt1) expression in benign and malignant melanocytic proliferations. A: Dnmt1 protein expression in human melanocytes and melanoma cell lines (SKMel28, 1205LU, A375, MeWo, MV3, SbCl2, WM35, WM793, WM1346, WM1366, WM1552, and WM115). B: DNMT1 RNA expression in nevi and primary melanomas. ${ }^{* *} P<0.01,{ }^{* * *} P<0.001$ (U-test). for each individual tumor. Epidermal progenitor cells in the basal and suprabasal layers of the epidermis were used as an internal positive control for the quantification of Dnmt1- and Ki-67-positive cells. ${ }^{19}$

\section{Patients}

FFPE tissue was retrieved from the archives of the Department of Dermatology and Institute of Pathology, University Hospital Tübingen (Tübingen, Germany). Dnmt1 and Ki-67 expression levels were analyzed in 13 nevi, 35 primary melanomas, and 3 melanoma metastases on a tissue microarray. A total of 32 metastases with known $B R A F-$ mutation status and 12 matched metastases (before treatment, $n=6$; time of progression/response, $n=6$ ) from six patients treated with the B-Rafi vemurafenib or the B-Rafi/ MEKi combination vemurafenib/cobimetinib were investigated. The study protocol was reviewed and approved by the ethics committee of the University Hospital Tübingen (project number 888/2017BO2).

\section{Results}

\section{Dnmt1 Expression in Nevi versus Melanoma}

On comparison of Dnmt1 protein expression in melanoma cell lines $(n=12)$ versus melanocytes isolated from human foreskin samples $(n=3)$, Dnmtl expression was significantly higher in the melanoma cell lines $[$ Dnmt $1 / \beta$-actin ratios (IQR), 0.12 (0.10 to 0.21 ) versus 0.06 (0.02 to 0.08 ); $P=0.009$ ( $U$-test)] (Figure 1A). To validate this finding in melanocytic tumors, data from available microarray data sets from 18 benign nevi and 45 primary melanomas were analyzed (Figure 1B). DNMT1 RNA expression was upregulated in the melanomas as compared with the nevi $[0.19(-0.02$ to 0.38$)$ versus $-0.47(-0.75$ to -0.05$)(\log 2$ centered); $P<0.001$ ( $U$-test)].

Correlations between Dnmt1 Expression and Melanoma Progression and Proliferation Rate in Melanocytic Tumors

As Dnmt1 is the key enzyme determining DNA methylation after cellular replication, the expression of Dnmt1 during melanoma progression and its association with cellular proliferation were explored. The expression levels of Dnmt 1 and $\mathrm{Ki}-67$ in melanocytes from FFPE nevi $(n=13)$, thin primary melanomas [median (IQR) Breslow thickness, 0.67 $\mathrm{mm}(0.47$ to 0.86$) ; n=35$ ], and metastases $(n=35)$ were first determined (Figure 2).

Dnmt1 protein levels increased incrementally from nevi to primary melanomas and metastatic melanomas, almost uniformly showing a strong expression [median (IQR) mean fluorescence intensity, 48.1 (41.7 to 59.6), 68.8 (58.4 to 77.0), and 87.5 (77.1 to 114.5), respectively] (Figure 2, A and B). A similar trend was found in the protein expression of the 

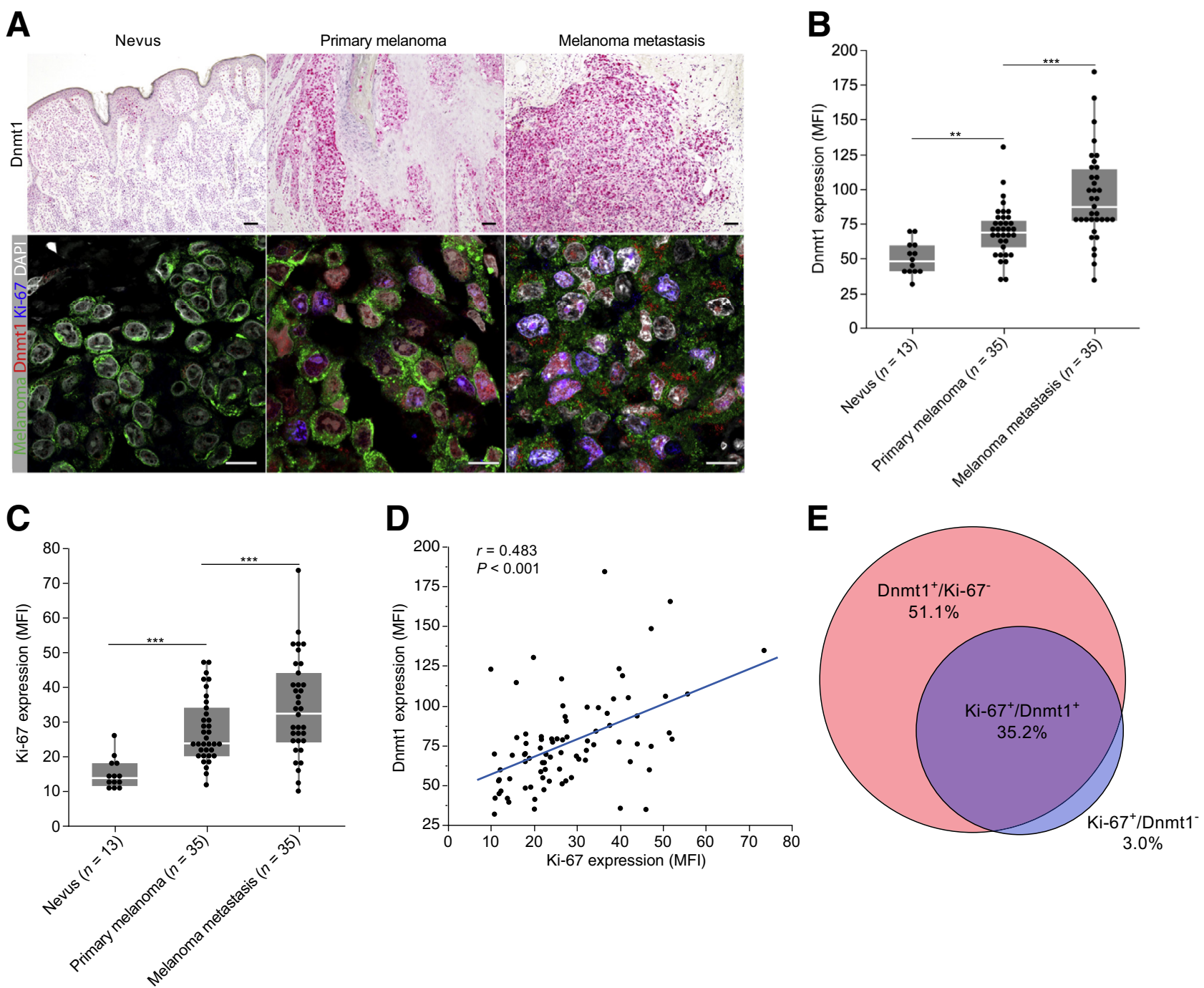

Figure 2 DNA methyltransferase 1 (Dnmt1) expression increases with melanoma progression and correlates with the proliferation marker Ki-67. A: Representative immunohistochemistry (top) and immunofluorescence staining (bottom) in nevus, primary melanoma, and melanoma metastasis. B and C: Mean fluorescence intensity (MFI) for Dnmt1 (B) and Ki-67 (C) in nevus, primary melanoma, and melanoma metastasis. D: Spearman correlation between Dnmt1 and Ki-67 expression. The linear regression line is shown in blue. E: Venn diagram depicting the percentages of Dnmt1- and Ki-67-positive cells in all analyzed melanocytic tumors. $n=83$ tumors (E). ${ }^{* *} P<0.01,{ }^{* *} P<0.001$ (U-test). Scale bars: $100 \mu \mathrm{m}$ (A, top row); $10 \mu \mathrm{m}(\mathbf{A}$, bottom row).

proliferation marker Ki-67, which was increased from nevi to primary melanomas and metastases [mean fluorescence intensity, 13.8 (11.9 to 18.0$), 23.6$ (20.1 to 33.9$)$, and 32.4 (24.1 to 44.0 ), respectively] (Figure 2C) and showed a strong positive correlation with Dnmt1 expression (Spearman correlation, $0.483 ; P<0.001$ ) (Figure 2D). At the single-cell level, most $(92.1 \%) \mathrm{Ki}-67-$ positive melanocytes were also positive for Dnmt1 (Figure 2E).

\section{Association between Dnmt1 Expression and Response to B-Raf and MEK Inhibition}

It has been shown that the expression of Dnmt1 is downregulated by knockdown of $B R A F \mathrm{~V} 600 \mathrm{E}$ in melanoma cell lines. ${ }^{20}$ Therefore, the Dnmt1 and Ki-67 expression levels in
$B R A F$-mutant and $B R A F$-wild-type melanoma were analyzed. Both Dnmt1 expression [median (IQR) mean fluorescence intensity, 95.9 (78.8 to 115.7 ) versus 86.1 (78.1 to 112.3); $P=0.55$ ] and Ki-67 expression [37.1 (24.1 to 45.7 ) versus 28.0 (24.0 to 42.3 ); $P=0.69$ ] were similar between $B R A F$-mutant and $B R A F$-wild-type metastatic melanoma ( $U$-test) (Figure 3, A and B).

To determine whether Dnmt1 expression could be modulated by pharmacologic inhibition of the mitogenactivated protein kinase (MAPK) pathway, BRAF-mutant melanoma cell lines were treated with the B-Rafi vemurafenib and/or the MEKi cobimetinib in vitro. With both drugs, Dnmt1 and the proliferation marker PCNA were markedly down-regulated in B-Rafi-sensitive but not in B-Rafi-resistant cell lines (Figure 3, C-E). 
A

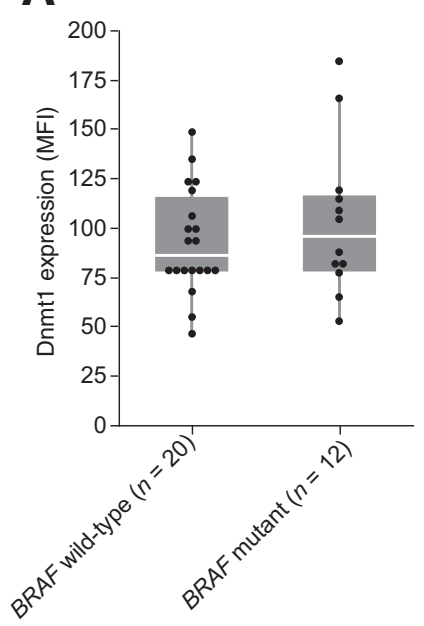

B

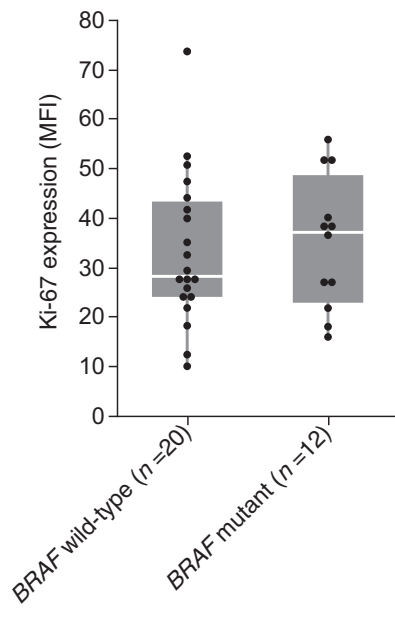

C

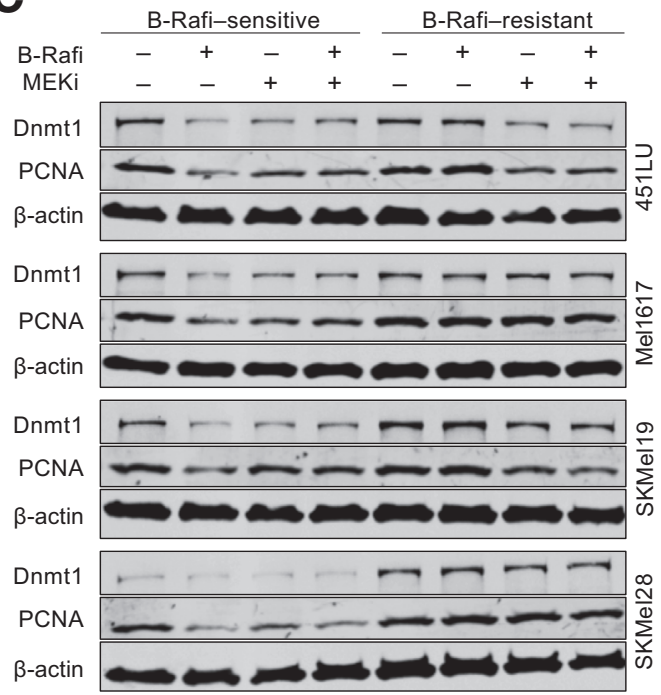

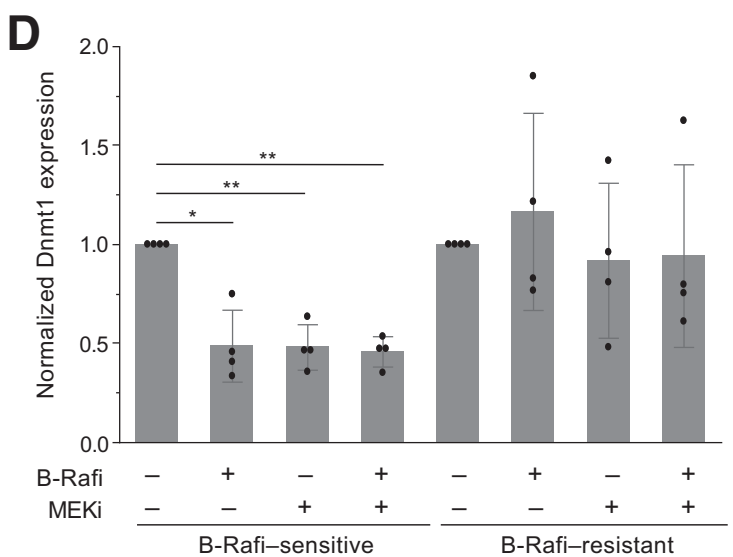

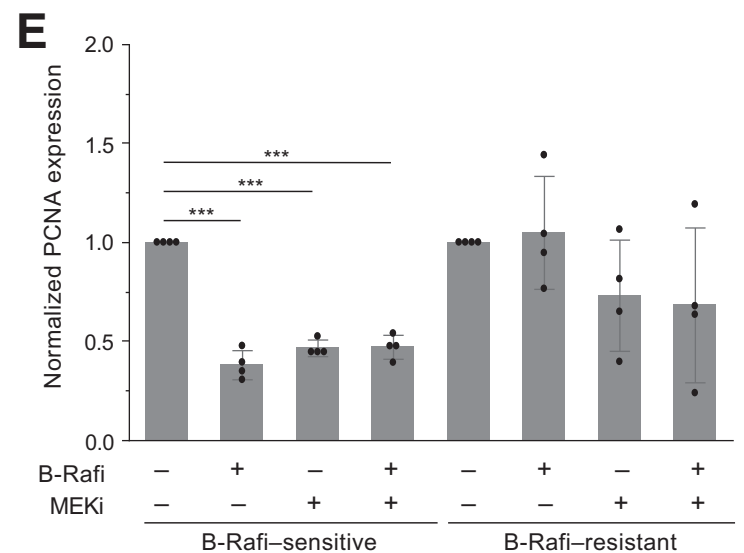

Figure 3 DNA methyltransferase 1 (Dnmt1) expression is independent of the BRAF-mutation status and depends on the mitogen-activated protein kinase pathway in BRAF-mutant melanoma cell lines. A and B: Mean fluorescence intensity (MFI) of Dnmt1 (A) and Ki-67 (B) in BRAF-wild-type and BRAF-mutant metastases. C-E: B-Raf inhibitor (B-Rafi)-sensitive and -resistant human melanoma cell lines 451LU, Mel1617, SKMel19, and SKMel28 were treated with the B-Rafi vemurafenib $(2 \mu \mathrm{mol} / \mathrm{L})$ and/or the mitogen-activated protein kinase inhibitor (MEKi) cobimetinib $(0.1 \mu \mathrm{mol} / \mathrm{L})$ for 24 hours. Subsequently, cell lysates were prepared and the protein levels of Dnmt1 and proliferating cell nuclear antigen (PCNA) were analyzed by Western blot analysis (C) and quantified by densitometry ( $\mathbf{D}$ and $\mathbf{E})$. $\beta$-actin served as loading control. Data are expressed as means \pm SD relative to dimethyl sulfoxide-treated cell lines from four independent experiments. ${ }^{*} P<0.05,{ }^{*} P>0.01$, and ${ }^{* * *} P<0.001$ (unpaired $t$-test).

To explore whether B-Rafi and MEKi also affect Dnmt1 expression in vivo, single-cell transcriptome data published by Rambow et $\mathrm{al}^{18}$ were analyzed. In that study, patientderived xenograft models from patients with $B R A F$ $\mathrm{V} 600 \mathrm{E} / \mathrm{K}$ - mutant melanoma were exposed to B-Raf and MEK inhibition. In brief, 250,000 melanoma cells were injected s.c. into 8- to 16-week-old female NMRI nude mice. Mice with tumors reaching $1000 \mathrm{~mm}^{3}$ were treated daily with the B-Rafi/MEKi combination dabrafenib/trametinib, and tumor volume was monitored with a caliper. All treated lesions shrank (phase 1, day 4) and reached an impalpable size (phase 2, day 28). However, continuous treatment led to the development of drug resistance and regrowth of the implanted tumors (phase 3, day 57). Tumor cells from each phase were isolated, and single-cell RNA sequencing of 674 cells was performed. Single-cell transcriptomics allowed for the analysis of DNMT1 and PCNA expression under treatment. A significant down-regulation of DNMT1 was induced in phases 1 and 2, followed by an up-regulation in the resistance phase 3 (Figure 4A). The same pattern was observed in six patients with Stage IV melanoma treated with targeted therapy in the Department of Dermatology, University of Tübingen (Figure 4B). All of the patients with progression on vemurafenib treatment $(n=3)$ showed a strong expression of DNMT1 before treatment and at the time of progression. In contrast, all patients with a response to vemurafenib/cobimetinib therapy $(n=3)$ showed a strong expression of DNMT1 before 


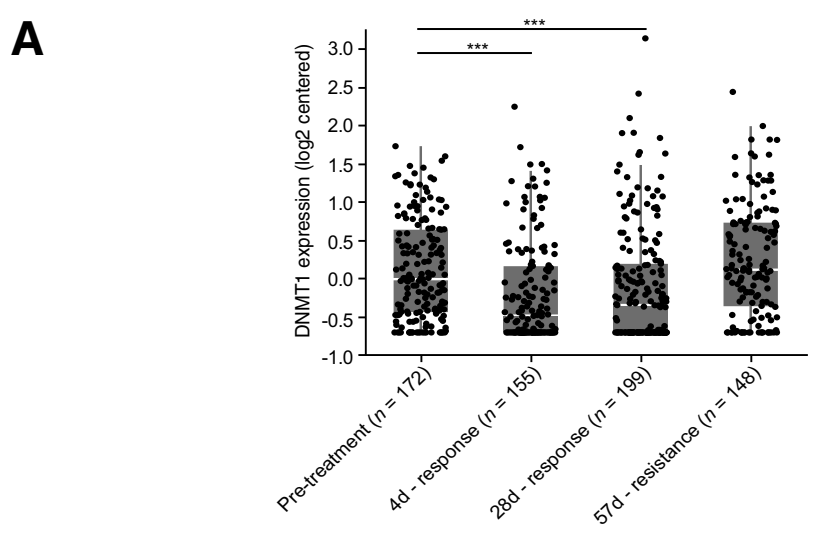

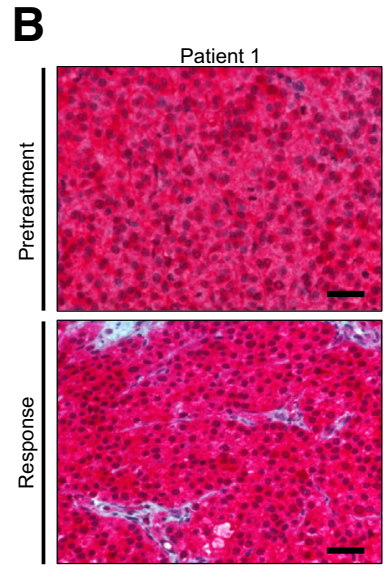

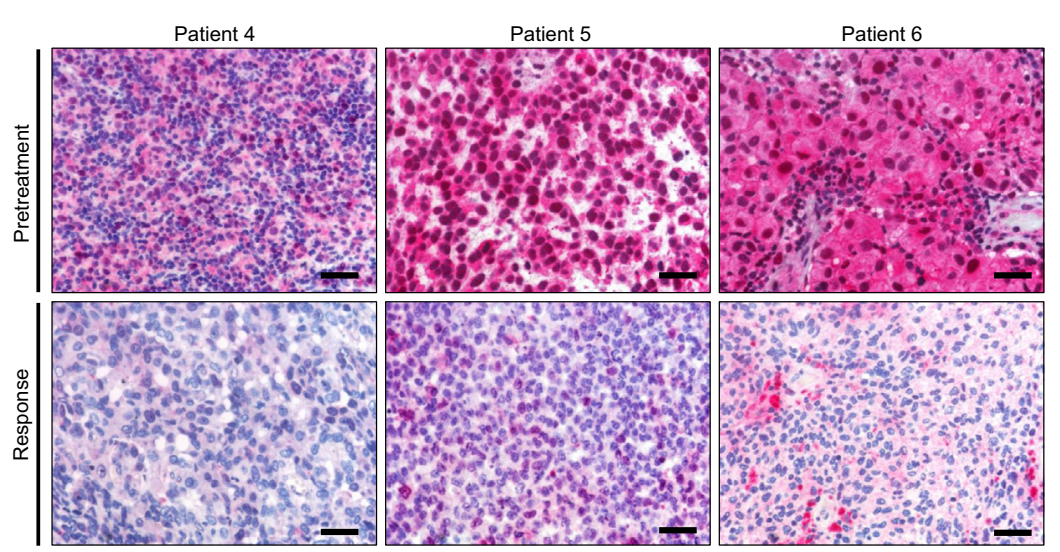

\section{C}

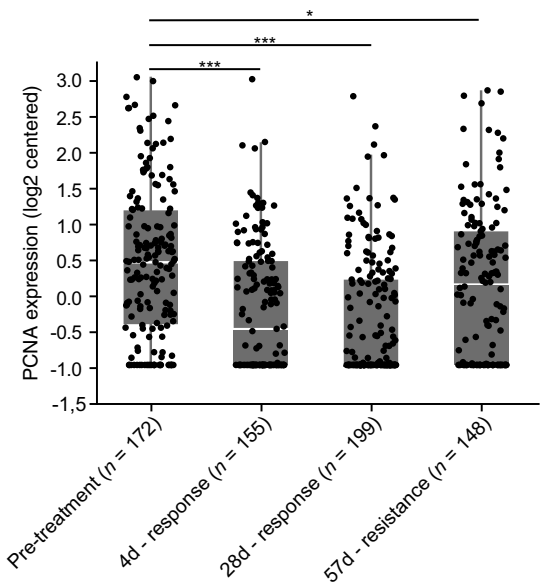

Figure 4 DNA methyltransferase 1 (Dnmt1) expression is associated with response to B-Raf and mitogen-activated protein kinase kinase (MEK) inhibition in vivo. A and C: DNMT1 (A) and PCNA (C) RNA expression in patient-derived xenograft models under treatment with the B-Raf/MEK inhibitor combination dabrafenib/trametinib. Target gene expression was determined by single-cell transcriptomics in a total of 674 cells before treatment and after 4 days (early response), 28 days (late response), and 57 days (resistance). B: Immunohistochemistry staining of Dnmt1 in matched metastases of patients treated with the B-Raf inhibitor vemurafenib (Patients 1 to 3 ) or the B-Raf/MEK inhibitor combination vemurafenib/cobimetinib (Patients 4 to 6 ). ${ }^{*} P<0.05, * * * P<0.001$ (Kruskal-Wallis test with post hoc Dunn-Bonferroni method). Scale bars $=100 \mu \mathrm{m}$.

treatment but a marked decrease at the time of response. The xenograft model also showed that the DNMT1 expression started to increase again earlier (phase 2, day 28) than did PCNA expression (phase 3, day 57) (Figure 4C). This finding suggests that the DNMT1 expression precedes the proliferative activity in melanoma and is not the secondary result of the proliferative state.
Human Melanoma Cells Resistant to B-Raf Inhibitors as Targets of Histone Deacetylase Inhibitor-Mediated Dnmt1 Degradation

It has been shown that histone deacetylase inhibitor increases Dnmt1 acetylation and stimulates its degradation. ${ }^{21}$ To investigate whether Dnmt1 is essential for the survival of melanoma 

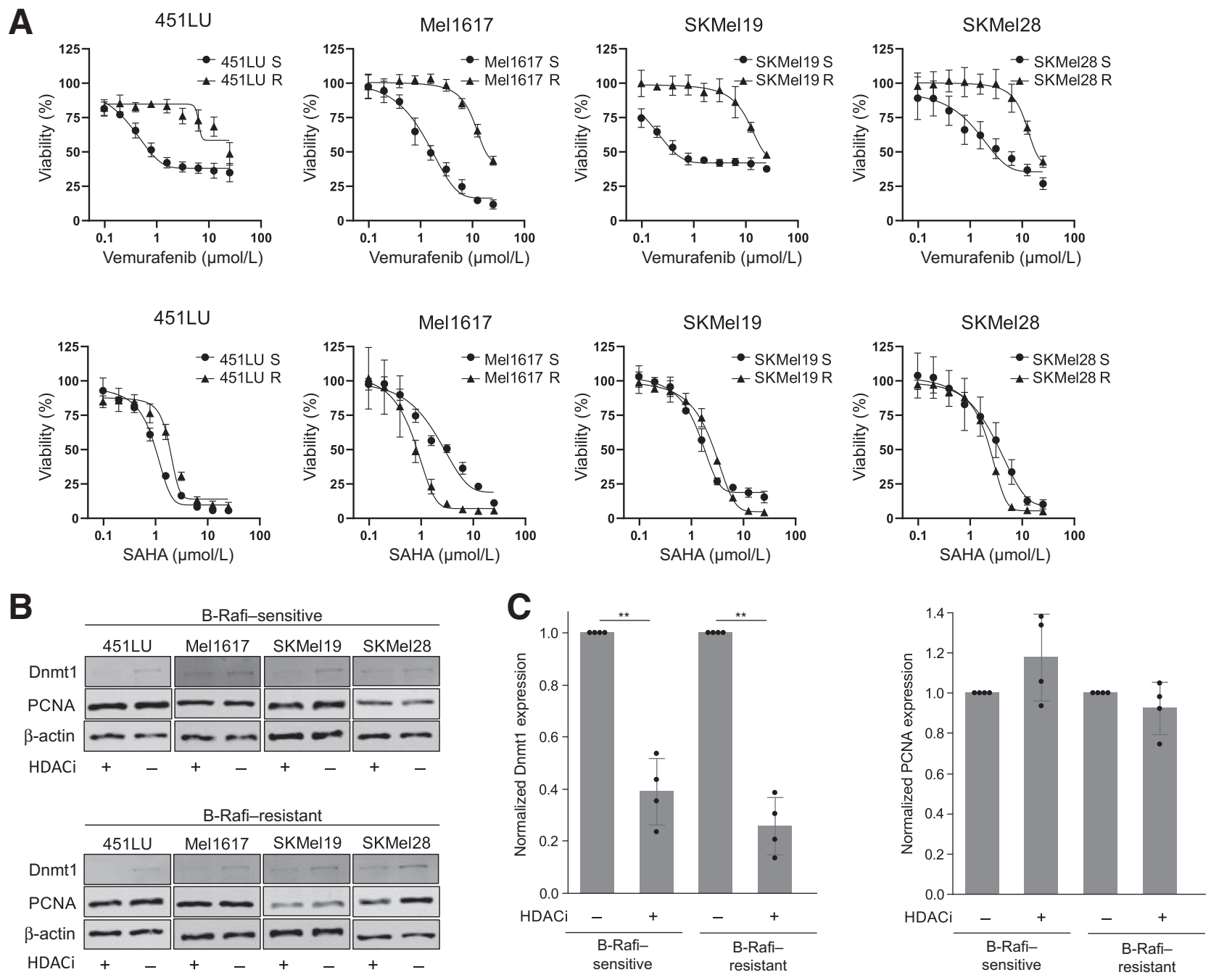

Figure 5 Human melanoma cells resistant to B-Raf inhibitor (B-Rafi) can be effectively targeted by histone deacetylase inhibitor (HDACi)-mediated DNA methyltransferase 1 (Dnmt1) degradation. A: Cell viability of B-Rafi-sensitive (S) and -resistant (R) cell lines 451LU, Mel1617, SKMel19, and SKMel28 was assessed with the 4-methylumbelliferyl heptanoate assay 72 hours after treatment with increasing concentrations of vemurafenib or suberoylanilide hydroxamic acid (SAHA). B and C: B-Rafi-sensitive and -resistant human melanoma cell lines were treated with the histone deacetylase inhibitor SAHA for 24 hours. Subsequently, cell lysates were prepared and the protein levels of Dnmt1 and proliferating cell nuclear antigen (PCNA) were analyzed by Western blot analysis and quantified by densitometry. $\beta$-actin served as a loading control. Data are expressed as the means \pm SD of six replicates relative to dimethyl sulfoxide-treated cell lines $(\mathbf{A})$ and relative to dimethyl sulfoxide-treated cell lines from four independent experiments (B and $\mathbf{C}) .{ }^{* *} P<0.01$ (unpaired $t$-test).

cells, B-Rafi-sensitive and -resistant cell lines were treated with the histone deacetylase inhibitor SAHA, and cell viability was measured after 72 hours (Figure 5A). SAHA was associated with impaired cell viability in both B-Rafi-sensitive and - resistant cell lines in a dose-dependent manner. Histone deacetylase inhibitor-mediated Dnmt1 down-regulation was not the consequence of reduced proliferation, as a significant Dnmt1 degradation was observed after 24 hours, when PCNA protein levels were still unchanged (Figure 5, B and C).

\section{Discussion}

Dnmt1 maintains methylation patterns after cellular replication and is essential for progenitor cell function and enriched in undifferentiated cells, where it is required for retaining proliferative stamina and the suppression of differentiation. ${ }^{19}$ In line with this finding, Dnmt1 expression was increased in primary and metastatic melanoma, and the correlation between Dnmt1 and the proliferation marker Ki-67 was strong. The close link between Dnmt1 and Ki-67 raises an important question: Is the up-regulation of Dnmt1 of pathogenic relevance for melanoma progression, or the mere reflection of an increased proliferation rate? Studies have reported that Dnmt1 was needed during the $S$ phase in order to maintain the methylation patterns of the newly synthesized strand, but that it is additionally associated with transcription factors during the $\mathrm{G}_{0} / \mathrm{G}_{1}$ and $\mathrm{G}_{2} / \mathrm{M}$ phases. ${ }^{22-24}$ In 1991, El-Deiry et $\mathrm{al}^{25}$ described that increased Dnmt1 expression characterized 
human cancer cells and may play a role in the pathogenesis of colon cancer. These findings were disputed a few years later by a group that reported normalized Dnmt1 expression to an $\mathrm{S}$ phase-specific proliferation marker and concluded that increased DNMT1 mRNA levels in colon cancer are nonspecific and reflect increased cell proliferation. ${ }^{26}$

However, more recent publications have demonstrated several proliferation-independent roles of Dnmt1 in cancer cells: Epigenetic editing of gene expression by Dnmt 1 can help tumor cells to escape attacks from the immune system, and it has been shown that Dnmt1 depletion in melanoma and renal cell carcinoma can overcome resistance to interferon-induced apoptosis. ${ }^{11}$ Moreover, the inhibition of Dnmt1 and DNA methylation has been reported to render tumors more immunogenic by increasing both cancer testis antigens and antigen presentation. ${ }^{11,27,28}$ Recent studies have also shown that Dnmt inhibitors induce an interferon response in cancer cells by activating double-stranded RNA sensors and reactivating viral defense genes, thus improving response to immunotherapy. ${ }^{29,30}$ A pathogenic role of Dnmt1 during melanoma progression was found by Venza et al, ${ }^{31}$ who reported that epigenetic down-regulation of E-cadherin through promotor hypermethylation was commonly found in melanoma and was associated with higher proliferation, metastasis, and poorer survival. Silencing of Dnmt1 but not Dnmt3a or Dnmt3b by siRNA was reported to restore E-cadherin expression in melanoma cell lines, and melanoma invasiveness was markedly inhibited with DNA hypomethylation. It has also been shown that a genetic DNMT1 polymorphism was associated with ulceration, nodal status, disease stage, progression, and poorer survival in melanoma patients. ${ }^{32}$

Treatment with histone deacetylase inhibitor has been associated with increased acetylated Dnmt1 and degradation of the protein. ${ }^{21}$ The data from the present study suggest that Dnmt1 not only is associated with proliferation in melanocytic tumors but also may be important for cell survival. Lombard et $\mathrm{al}^{33}$ and Maertens et $\mathrm{al}^{34}$ recently reported that SAHA potentiated the effect of B-Rafi/MEKi in BRAF-mutant melanoma cells and that histone deacetylase inhibition was associated with profound tumor regression, even in a tumor model that was resistant to B-Rafi/MEKi treatment. Down-regulation of Dnmt1 has also been achieved with the demethylating agents azacitidine and decitabine, which have been approved for use in the treatment of hematologic malignancies. ${ }^{35-37}$ These hypomethylating drugs are being studied in patients with metastatic melanoma and have recently shown promising immunomodulatory and antitumor activity. ${ }^{7,38} B R A F$ V600E is the most common driver mutation in cutaneous melanoma and has been reported to promote aberrant gene methylation by the overexpression of Dnmt1 and enhancer of zeste homolog $2 .^{20}$ Dnmt1 is down-regulated by knockdown of BRAF V600E in melanoma cell lines, and it has been shown in a series of nonmelanoma cancer cell lines that the MEK/ERK pathway was essential for the overexpression of Dnmt $1 .{ }^{39}$ Our results add to the growing body of evidence that Dnmt1 expression depends on the MAPK pathway in cutaneous melanoma. However, the epigenetic impact of targeted therapies on melanoma cells is unclear at the moment and remains to be defined in future studies.

In conclusion, the findings from the present study show, for the first time, a correlation between Dnmt1 expression and cellular proliferation in melanocytic tumors. Given its strong expression in metastatic melanoma, as well as its known immunomodulatory role and association with the response to B-Rafi/MEKi, Dnmt1 may be a promising target in combination with immunotherapy or targeted therapy.

\section{Acknowledgments}

We thank Renate Nordin, Monika Beer, Letehawariat Haile, Sybille Kohler, Angelika Koj-Kurzynski, Petra Leber, Margareta Leippert, Simon Riel, and Karen Greif for excellent technical assistance and Ulrike Keim for statistical advice.

\section{Supplemental Data}

Supplemental material for this article can be found at https://doi.org/10.1016/j.ajpath.2020.07.002.

\section{References}

1. Sharma S, Kelly TK, Jones PA: Epigenetics in cancer. Carcinogenesis 2010, 31:27-36

2. Roh MR, Gupta S, Park KH, Chung KY, Lauss M, Flaherty KT, Jonsson G, Rha SY, Tsao H: Promoter methylation of PTEN is a significant prognostic factor in melanoma survival. J Invest Dermatol 2016, 136:1002-1011

3. Conway K, Edmiston SN, Khondker ZS, Groben PA, Zhou X, Chu H, Kuan PF, Hao H, Carson C, Berwick M, Olilla DW, Thomas NE: DNA-methylation profiling distinguishes malignant melanomas from benign nevi. Pigment Cell Melanoma Res 2011, 24:352-360

4. Gao L, Smit MA, van den Oord JJ, Goeman JJ, Verdegaal EM, van der Burg SH, Stas M, Beck S, Gruis NA, Tensen CP, Willemze R, Peeper DS, van Doorn R: Genome-wide promoter methylation analysis identifies epigenetic silencing of MAPK13 in primary cutaneous melanoma. Pigment Cell Melanoma Res 2013, 26:542-554

5. Micevic G, Theodosakis N, Bosenberg M: Aberrant DNA methylation in melanoma: biomarker and therapeutic opportunities. Clin Epigenetics 2017, 9:34

6. Bestor TH: Activation of mammalian DNA methyltransferase by cleavage of a $\mathrm{Zn}$ binding regulatory domain. EMBO J 1992, 11: 2611-2617

7. Di Giacomo AM, Covre A, Finotello F, Rieder D, Danielli R, Sigalotti L, Giannarelli D, Petitprez F, Lacroix L, Valente M, Cutaia O, Fazio C, Amato G, Lazzeri A, Monterisi S, Miracco C, Coral S, Anichini A, Bock C, Nemc A, Oganesian A, Lowder J, Azab M, Fridman WH, Sautes-Fridman C, Trajanoski Z, Maio M: Guadecitabine plus ipilimumab in unresectable melanoma: the NIBIT-M4 clinical trial. Clin Cancer Res 2019, 25:7351-7362

8. Cruickshanks HA, McBryan T, Nelson DM, Vanderkraats ND, Shah PP, van Tuyn J, Singh Rai T, Brock C, Donahue G, Dunican DS, Drotar ME, Meehan RR, Edwards JR, Berger SL, 
Adams PD: Senescent cells harbour features of the cancer epigenome. Nat Cell Biol 2013, 15:1495-1506

9. Fournel M, Sapieha P, Beaulieu N, Besterman JM, MacLeod AR: Down-regulation of human DNA-(cytosine-5) methyltransferase induces cell cycle regulators p16(ink4A) and p21(WAF/Cip1) by distinct mechanisms. J Biol Chem 1999, 274:24250-24256

10. Robert MF, Morin S, Beaulieu N, Gauthier F, Chute IC, Barsalou A, MacLeod AR: DNMT1 is required to maintain CpG methylation and aberrant gene silencing in human cancer cells. Nat Genet 2003, 33: $61-65$

11. Reu FJ, Bae SI, Cherkassky L, Leaman DW, Lindner D, Beaulieu N, MacLeod AR, Borden EC: Overcoming resistance to interferoninduced apoptosis of renal carcinoma and melanoma cells by DNA demethylation. J Clin Oncol 2006, 24:3771-3779

12. Muller-Hermelink N, Braumuller $\mathrm{H}$, Pichler B, Wieder $\mathrm{T}$, Mailhammer R, Schaak K, Ghoreschi K, Yazdi A, Haubner R, Sander CA, Mocikat R, Schwaiger M, Forster I, Huss R, Weber WA, Kneilling M, Rocken M: TNFR1 signaling and IFN-gamma signaling determine whether $\mathrm{T}$ cells induce tumor dormancy or promote multistage carcinogenesis. Cancer Cell 2008, 13:507-518

13. Braumuller $\mathrm{H}$, Wieder $\mathrm{T}$, Brenner $\mathrm{E}$, Assmann $\mathrm{S}$, Hahn $\mathrm{M}$, Alkhaled M, Schilbach K, Essmann F, Kneilling M, Griessinger C, Ranta F, Ullrich S, Mocikat R, Braungart K, Mehra T, Fehrenbacher B, Berdel J, Niessner H, Meier F, van den Broek M, Haring HU, Handgretinger R, Quintanilla-Martinez L, Fend F, Pesic M, Bauer J, Zender L, Schaller M, Schulze-Osthoff K, Rocken M: T-helper-1-cell cytokines drive cancer into senescence. Nature 2013, 494:361-365

14. Brenner E, Schorg BF, Ahmetlic F, Wieder T, Hilke FJ, Simon N, Schroeder C, Demidov G, Riedel T, Fehrenbacher B, Schaller M, Forschner A, Eigentler T, Niessner H, Sinnberg T, Bohm KS, Homberg N, Braumuller H, Dauch D, Zwirner S, Zender L, Sonanini D, Geishauser A, Bauer J, Eichner M, Jarick KJ, Beilhack A, Biskup S, Docker D, Schadendorf D, Quintanilla-Martinez L, Pichler BJ, Kneilling M, Mocikat R, Rocken M: Cancer immune control needs senescence induction by interferon-dependent cell cycle regulator pathways in tumours. Nat Commun 2020, 11:1335

15. Sinnberg T, Makino E, Krueger MA, Velic A, Macek B, Rothbauer U, Groll N, Potz O, Czemmel S, Niessner H, Meier F, Ikenberg K, Garbe C, Schittek B: A nexus consisting of beta-catenin and Stat3 attenuates BRAF inhibitor efficacy and mediates acquired resistance to vemurafenib. EBioMedicine 2016, 8:132-149

16. Mancianti ML, Herlyn M, Weil D, Jambrosic J, Rodeck U, Becker D, Diamond L, Clark WH, Koprowski H: Growth and phenotypic characteristics of human nevus cells in culture. J Invest Dermatol 1988, 90: 134-141

17. Talantov D, Mazumder A, Yu JX, Briggs T, Jiang Y, Backus J, Atkins D, Wang Y: Novel genes associated with malignant melanoma but not benign melanocytic lesions. Clin Cancer Res 2005, 11:7234-7242

18. Rambow F, Rogiers A, Marin-Bejar O, Aibar S, Femel J, Dewaele M, Karras P, Brown D, Chang YH, Debiec-Rychter M, Adriaens C, Radaelli E, Wolter P, Bechter O, Dummer R, Levesque M, Piris A, Frederick DT, Boland G, Flaherty KT, van den Oord J, Voet T, Aerts S, Lund AW, Marine JC: Toward minimal residual diseasedirected therapy in melanoma. Cell 2018, 174:843-55 e19

19. Sen GL, Reuter JA, Webster DE, Zhu L, Khavari PA: DNMT1 maintains progenitor function in self-renewing somatic tissue. Nature 2010, 463:563-567

20. Hou P, Liu D, Dong J, Xing M: The BRAF(V600E) causes widespread alterations in gene methylation in the genome of melanoma cells. Cell Cycle 2012, 11:286-295

21. Cheng J, Yang H, Fang J, Ma L, Gong R, Wang P, Li Z, Xu Y: Molecular mechanism for USP7-mediated DNMT1 stabilization by acetylation. Nat Commun 2015, 6:7023

22. Sharif J, Muto M, Takebayashi S, Suetake I, Iwamatsu A, Endo TA, Shinga J, Mizutani-Koseki Y, Toyoda T, Okamura K, Tajima S,
Mitsuya K, Okano M, Koseki H: The SRA protein Np95 mediates epigenetic inheritance by recruiting Dnmt1 to methylated DNA. Nature 2007, 450:908-912

23. Easwaran HP, Schermelleh L, Leonhardt H, Cardoso MC: Replication-independent chromatin loading of Dnmt1 during G2 and M phases. EMBO Rep 2004, 5:1181-1186

24. Hervouet E, Nadaradjane A, Gueguen M, Vallette FM, Cartron PF: Kinetics of DNA methylation inheritance by the Dnmt1-including complexes during the cell cycle. Cell Div 2012, 7:5

25. El-Deiry WS, Nelkin BD, Celano P, Yen RW, Falco JP, Hamilton SR, Baylin SB: High expression of the DNA methyltransferase gene characterizes human neoplastic cells and progression stages of colon cancer. Proc Natl Acad Sci U S A 1991, 88:3470-3474

26. Lee PJ, Washer LL, Law DJ, Boland CR, Horon IL, Feinberg AP: Limited up-regulation of DNA methyltransferase in human colon cancer reflecting increased cell proliferation. Proc Natl Acad Sci U S A 1996, 93:10366-10370

27. Siebenkas C, Chiappinelli KB, Guzzetta AA, Sharma A, Jeschke J, Vatapalli R, Baylin SB, Ahuja N: Inhibiting DNA methylation activates cancer testis antigens and expression of the antigen processing and presentation machinery in colon and ovarian cancer cells. PLoS One 2017, 12:e0179501

28. Haffner MC, Taheri D, Luidy-Imada E, Palsgrove DN, Eich ML, Netto GJ, Matoso A, Nirschl TR, Zheng Q, Hicks JL, Nelson WG, De Marzo AM, Marchionni L, Drake CG, Yegnasubramanian S: Hypomethylation, endogenous retrovirus expression, and interferon signaling in testicular germ cell tumors. Proc Natl Acad Sci U S A 2018, 115:E8580-E8582

29. Chiappinelli KB, Strissel PL, Desrichard A, Li H, Henke C, Akman B, Hein A, Rote NS, Cope LM, Snyder A, Makarov V, Budhu S, Slamon DJ, Wolchok JD, Pardoll DM, Beckmann MW, Zahnow CA, Merghoub T, Chan TA, Baylin SB, Strick R: Inhibiting DNA methylation causes an interferon response in cancer via dsRNA including endogenous retroviruses. Cell 2015, 162:974-986

30. Luo N, Nixon MJ, Gonzalez-Ericsson PI, Sanchez V, Opalenik SR, Li H, Zahnow CA, Nickels ML, Liu F, Tantawy MN, Sanders ME, Manning HC, Balko JM: DNA methyltransferase inhibition upregulates MHC-I to potentiate cytotoxic T lymphocyte responses in breast cancer. Nat Commun 2018, 9:248

31. Venza M, Visalli M, Catalano T, Biondo C, Beninati C, Teti D, Venza I: DNA methylation-induced E-cadherin silencing is correlated with the clinicopathological features of melanoma. Oncol Rep 2016, 35:2451-2460

32. Maric H, Supic G, Kandolf-Sekulovic L, Maric V, Mijuskovic Z, Radevic T, Rajovic M, Magic Z: DNMT1 and DNMT3B genetic polymorphisms affect the clinical course and outcome of melanoma patients. Melanoma Res 2019, 29:596-602

33. Lombard DB, Cierpicki T, Grembecka J: Combined MAPK pathway and HDAC inhibition breaks melanoma. Cancer Discov 2019, 9:469-471

34. Maertens O, Kuzmickas R, Manchester HE, Emerson CE, Gavin AG, Guild CJ, Wong TC, De Raedt T, Bowman-Colin C, Hatchi E, Garraway LA, Flaherty KT, Pathania S, Elledge SJ, Cichowski K: MAPK pathway suppression unmasks latent DNA repair defects and confers a chemical synthetic vulnerability in BRAF-, NRAS-, and NF1-mutant melanomas. Cancer Discov 2019, 9:526-545

35. Liu B, Song J, Luan J, Sun X, Bai J, Wang H, Li A, Zhang L, Feng X, Du Z: Promoter methylation status of tumor suppressor genes and inhibition of expression of DNA methyltransferase 1 in non-small cell lung cancer. Exp Biol Med (Maywood) 2016, 241:1531-1539

36. Kaminskas E, Farrell A, Abraham S, Baird A, Hsieh LS, Lee SL, Leighton JK, Patel H, Rahman A, Sridhara R, Wang YC, Pazdur R: FDA: approval summary: azacitidine for treatment of myelodysplastic syndrome subtypes. Clin Cancer Res 2005, 11:3604-3608

37. Chiappinelli KB, Zahnow CA, Ahuja N, Baylin SB: Combining epigenetic and immunotherapy to combat cancer. Cancer Res 2016, $76: 1683-1689$ 
38. Burton EM, Woody T, Glitza IC, Amaria RN, Keung EZ, Diab A, Patel SP, Wong MKK, Yee C, Hwu P, McQuade JL, Woodman SE, Tetzlaff MT, Davies MA, Wargo JA, Rai K, Tawbi HA: A phase II study of oral azacitidine (CC-486) in combination with pembrolizumab (PEMBRO) in patients (pts) with metastatic melanoma (MM). J Clin Oncol 2019, 37, Abstract 9560

39. Li J, Wang R, Hu X, Gao Y, Wang Z, Li J, Wong J: Activated MEK/ERK pathway drives widespread and coordinated overexpression of UHRF1 and DNMT1 in cancer cells. Sci Rep 2019, 9:907 